\title{
General Relativity Histories Theory
}

\author{
Ntina Savvidou \\ Physics Department, Theory Group, Imperial College, \\ South Kensington, London SW72BZ, UK \\ and \\ Physics Department, University of Patras, 26500 Patras, Greece
}

Received on 20 December, 2004

\begin{abstract}
The canonical description is based on the prior choice of a spacelike foliation, hence making a reference to a spacetime metric. However, the metric is expected to be a dynamical, fluctuating quantity in quantum gravity. After presenting the developments in the History Projection Operator histories theory in the last seven years giving special emphasis on the novel temporal structure of the formalism - we show how this problem can be solved in the histories formulation of general relativity. We implement the $3+1$ decomposition using metricdependent foliations which remain spacelike with respect to all possible Lorentzian metrics. This allows us to find an explicit relation of covariant and canonical quantities which preserves the spacetime character of the canonical description. In this new construction we have a coexistence of the spacetime diffeomorphisms group Diff(M) and the Dirac algebra of constraints.
\end{abstract}

\section{INTRODUCTION}

In this work we present the application of ideas of consistent histories to general relativity, and its potential implications for the quantisation of the theory - regarding in particular the emphasis on spacetime concepts of the histories. We show how the temporal structure of the Histories Projection Operator formalism led to some very important consequences regarding the physical results of canonical general relativity.

One of the major approaches to the quantisation of gravity is the canonical one, either in its original form - involving geometrodynamic variables - or in terms of the loop variables, introduced via the connection formulation of canonical general relativity.

The canonical quantisation involves:

i) the identification of a Hilbert space on which the canonical commutation relations - or some other appropriate algebraic structure - can be implemented, thereby defining the kinematical variables of quantum gravity. The Hilbert space is chosen to allow the representation of the constraints of the Hamiltonian description in terms of self-adjoint operators, preserving the classical Dirac algebra of constraints.

ii) then, one has to find the zero eigenspace of the constraint operators, in order to define the physical Hilbert space. This is the scope of the original Dirac quantisation of constrained systems: variations are usually employed in the case of gravity (or special models), because the constraint operators are not expected to have a discrete spectrum.

However the canonical quantisation scheme suffers also from serious problems, both on technical level and conceptual level. For example, we encounter problems in constructing the Hilbert space, writing the constraint operators, and finding their spectrum. Also, the fact that general relativity is a generally covariant theory raises grave doubts about the conceptual adequacy of the canonical method of quantisation.

Furthermore, the equations of general relativity are covariant with respect to the action of the diffeomorphisms group $\operatorname{Diff}(M)$, of the spacetime manifold $M$. This does not pose great difficulties in the classical theory, since once the equa- tions of motion are solved the Lorentzian metric on $M$ can be used to implement concepts like causality and spacelike separation. In quantum theory however, such notions as causality and spacelike separation are lost, because the geometry of spacetime is expected to be subject to quantum fluctuations.

This creates problems even at the first step of the quantisation procedure, namely the definition of the canonical commutation relations. The canonical commutation relations are defined on a 'spacelike' surface, however, a surface is spacelike with respect to some particular spacetime metric $g$, which is itself a quantum observable that is expected to fluctuate.

The prior definability of the canonical commutation relations is not merely a mathematical requirement: in a generic quantum field theory the canonical commutation relations implement the principle of microcausality: namely that field observables that are defined in spacelike separated regions commute. However, if the notion of spacelikeness is also dynamical, it is not clear in what way this relation will persist.

A spacelike foliation is necessary for the implementation of the $3+1$ decomposition and the definition of the Hamiltonian. Again we are faced with the question of how to: reconcile the requirement of spacelikeness with the expectation that different metrics will take part in the quantum description.

Even more, one may question whether the predictions of the resulting quantum theories are independent of the choice of foliation. The Hilbert space of the quantum theory, which it is constructed canonically, is not straightforwardly compatible with the $\operatorname{Diff}(M)$ symmetry. In the canonical theory, the symmetry group is the one generated by the Dirac algebra of constraints, which is mathematically distinct from the $\operatorname{Diff}(M)$ group.

In effect, different choices of foliation lead a priori to different quantum theories and there is no guarantee that these quantum theories are unitarily equivalent (or physically equivalent in some other generalised sense).

The canonical description cannot provide an answer to these questions, because once the foliation is employed for the $3+1$ decomposition, its effect is lost, and there is no way of relating the predictions corresponding to different foliations.

The above are serious problems, which challenge the va- 
lidity of the canonical approach towards the description of a generally covariant theory of quantum gravity.

Finally, the problem which is perhaps most well known, is the problem of time. The Hamiltonian of general relativity is a combination of the first class constraints, hence it vanishes on the reduced state space. It is expected also to vanish on the physical Hilbert space constructed in the quantisation scheme. This means that there is no notion of time evolution in the space of true degrees of freedom. More than that, the notion of time as causal ordering seems to be lost. In contrast, the tensorial expressions of the equations of motion are $\operatorname{Diff}(M)$-invariant in the Lagrangian formalism. It seems very natural, therefore, to wish for a theory that combines the virtues of both formalisms: the Lagrangian, and the Hamiltonian. Such a theory is the history projection operatorhistories (HPO) scheme, which offers the possibility of handling the ideas of space and time in significantly new ways.

The consistent histories scheme was developed by Griffiths and Omnés [1] as an interpretation of quantum theory for closed systems. Gell-Mann and Hartle [2] elaborated this scheme in the case of quantum cosmology.

Further development came from Isham and Linden and collaborators in the HPO histories scheme [3-6], in which they were able to represent histories by projection operators on a suitable Hilbert space, thus emphasising the temporal quantum logic of the frame work.

An important feature of the HPO histories is the augmented temporal structure [7], which allows us to mathematically implement the distinction between time as a parameter of kinematics and as a parameter of dynamics. It is of great significance that, in the context of classical canonical general relativity this distinction provides a framework in which the spacetime diffeomorphism group coexists with the Dirac algebra of constraints. This is a very significant result: it implies that there is a central role for spacetime concepts, as opposed to the domination by spatial ideas in the normal canonical approaches to quantum gravity. More important, it allows a kinematical description in which different choices of the direction of time coexist, in a way that always preserves the spacetime character of the theory.

The (general relativity) constraints, depend on the foliation functional. This leads to the natural question, whether physical results depend upon this choice. The solution of the constraints determines a reduced phase space for histories, which has an explicit dependence on the foliation. In $[8,9]$ it was showed that the action of the spacetime diffeomorphism group intertwines between different reduction procedures. Moreover, if one requires that a specific physical 'equivariance condition' is satisfied by the foliation functional, then the reduced state space is invariant under the action of the diffeomorphism group. This is a completely novel result, which has been made possible only by the incorporation of general relativity into the histories formalism.

The histories approach to general relativity suggests a new, spacetime focussed, approach to quantum gravity that is characterised by two features that are not implemented in any of the existing, direct, quantum gravity schemes.
First, the Lorentzian metric is quantised, as a direct analogue of the way the 'external' quantum field arises in the history approach to scalar quantum field theory $[10,11]$. On the other hand, in the conventional canonical quantum gravity schemes only the spatial metric on a three-surface is quantised.

Second, the history scheme incorporates intrinsically the basic symmetry of general relativity, namely general covariance, as manifested by the existence of a realisation of the group of spacetime diffeomorphisms, and under whose action the history analogue of the canonical Hamiltonian constraints are invariant.

\section{CONSISTENT HISTORIES PRELIMINARY}

The consistent histories formalism was originally developed by Griffiths and Omnés [1], as an interpretation of quantum theory for closed systems.

Gell-Mann and Hartle [2] elaborated this scheme in the case of quantum cosmology - the Universe being regarded as a closed system. They emphasised in particular that a theory of quantum gravity that is expected to preserve the spacetime character of general relativity would need a quantum formalism in which the irreducible elements are temporally extended objects, namely histories.

The basic object in the consistent histories approach is a history

$$
\alpha:=\left(\hat{\alpha}_{t_{1}}, \hat{\alpha}_{t_{2}}, \ldots, \hat{\alpha}_{t_{n}}\right),
$$

which is a time-ordered sequence of properties of the physical system, each one represented by a single-time projection operator on the standard Hilbert space. We notice that the emphasis is given on histories rather than states at a single time.

The probabilities and the dynamics are contained in the decoherence functional, a complex-valued function on the space of histories

$$
d_{H, \rho}(\alpha, \beta)=\operatorname{tr}\left(\tilde{C}_{\alpha}^{\dagger} \rho_{t_{0}} C_{\beta}\right)
$$

where $\rho_{t_{0}}$ is the initial quantum state and where

$$
\tilde{C}_{\alpha}:=U\left(t_{0}, t_{1}\right) \hat{\alpha}_{t_{1}} U\left(t_{1}, t_{2}\right) \ldots U\left(t_{n-1}, t_{n}\right) \hat{\alpha}_{t_{n}} U\left(t_{n}, t_{0}\right)
$$

is the class operator that represents the history $\alpha$.

When a set of histories satisfies a decoherence condition,

$$
d_{\mathcal{H}, \rho}(\alpha, \beta)=0 \quad \text { then } \alpha, \beta \text { in the consistent set }
$$

which means that we have zero interference between different histories, then it is possible to consistently assign probabilities to each history in that set; it is called a consistent set.

Then we can assign probabilities to each history in the consistent set

$$
d_{\mathcal{H}, \rho}(\alpha, \alpha)=\operatorname{Prob}\left(\alpha ; \rho_{t_{0}}\right)=\operatorname{tr}\left(\tilde{C}_{\alpha}^{\dagger} \rho_{t_{0}} C_{\alpha}\right) .
$$




\section{HISTORY PROJECTION OPERATOR-BASIC ELEMENTS}

In the History Projection Operator(HPO) approach to consistent histories theory the emphasis is given on the temporal quantum logic.

A history is represented by a tensor product of projection operators

$$
\hat{\alpha}:=\hat{\alpha}_{t_{1}} \otimes \hat{\alpha}_{t_{2}} \otimes \ldots \otimes \hat{\alpha}_{t_{n}},
$$

each operator $\hat{\alpha}_{t_{i}}$ being defined on a copy of the single-time Hilbert space $\mathcal{H}_{t_{i}}$ at that time $t_{i}$ and corresponding to some property of the system at the same time indicated by the $\mathrm{t}$ label. Therefore a history is itself a genuine projection operator defined on the history Hilbert space $\mathcal{V}_{n}$, which is a tensor product of the single-time Hilbert spaces

$$
\mathcal{V}_{n}:=\mathcal{H}_{t_{1}} \otimes \mathcal{H}_{t_{2}} \otimes \ldots \otimes \mathcal{H}_{t_{n}} .
$$

In order to define continuous time histories, we do not take the continuous limit of the tensor product of Hilbert spaces, as it cannot be properly defined. The history group, which is a generalised analogue of the canonical group of standard quantum theory for elementary systems, was employed [5] in order to construct the continuous-time history Hilbert space.

For example, for a particle moving on a line the single-time canonical commutation relations

$$
\begin{aligned}
& {\left[\hat{x}, \hat{x}^{\prime}\right]=0=\left[\hat{p}, \hat{p}^{\prime}\right]} \\
& {[\hat{x}, \hat{p}]=i \hbar}
\end{aligned}
$$

become the history group that it is described by the following history commutation relations, defined at unequal moments of time

$$
\begin{aligned}
{\left[\hat{x}_{t}, \hat{x}_{t^{\prime}}\right] } & =0=\left[\hat{p}_{t}, \hat{p}_{t^{\prime}}\right] \\
{\left[\hat{x}_{t}, \hat{p}_{t^{\prime}}\right] } & =i \hbar \delta\left(t-t^{\prime}\right) .
\end{aligned}
$$

The key idea in the definition of the history group is, that, the spectral projectors of the generators of its Lie algebra represent propositions about phase space observables of the system.

The notion of a 'continuous tensor product' - and hence 'continuous temporal logic' - arises via a representation of the history algebra. In order to describe discrete-time histories we have to replace the delta function, on the right-hand side of Eq. (11), with the Kronecker delta.

Propositions about histories of the system are associated with projectors on history Hilbert space. We must clarify here that the operator $x_{t}$ refers to the position of the particle at a specific fixed moment of time $t$. As we shall see in the following section, the novel temporal structure that was later introduced [7], allowed the interpretation of the index $t$ as the index that does not refer to dynamics - it is not the parameter of time evolution - it is the label of the temporal quantum logic, in the sense that it refers to the time a proposition about momentum or position is asserted.

It is important to remark that physical quantities are naturally time-averaged in this scheme. The smeared form of the history algebra

$$
\begin{aligned}
& {\left[\hat{x}_{f}, \hat{x}_{g}\right]=0=\left[\hat{p}_{f}, \hat{p}_{g}\right]} \\
& {\left[\hat{x}_{f}, \hat{p}_{g}\right]=i \hbar(f, g),}
\end{aligned}
$$

where: $(f, g)=\int_{-\infty}^{\infty} d t f(t) g(t)$, resembles that of an onedimensional quantum field theory and therefore techniques from quantum field theory may be used in the study of these representations. Analogous versions of the history group have been studied for other field theories [10, 13].

The existence of a properly defined Hamiltonian operator $H$ is proved to uniquely select the physically appropriate representation of the history algebra, therefore the definition of the time-averaged energy operator $H$ is crucial for the formalism.

\section{HPO-TEMPORAL STRUCTURE}

In order to study the temporal structure of the HPO theory we use the model of a one-dimensional simple harmonic oscillator, however the results are generalised appropriately for other systems.

One of the crucial problems for the development of the HPO theory was the lack of a clear notion of time evolution, in the sense that, there was no natural way to express the time translations from one time slot - that refers to one copy of the Hilbert space $\mathcal{H}_{t}$ - to another one, that refers to another copy $\mathcal{H}_{t^{\prime}}$. The introduction of the history group allowed the definition of continuous-time histories and led to 'time-averaged' physical observables, however any notion of dynamics was lost and the theory was brought to a hold.

The situation changed after the introduction of a new idea concerning the notion of time: the distinction between dynamics and kinematics corresponds to the mathematical distinction between the notion of 'time evolution' from that of 'time ordering' or 'temporal logic time'. The distinction proved very fruitful for the development of the history theory, leading in particular to the results of general relativity.

The crucial step in the identification of the temporal structure of the theory was the definition in [10] of the action operator $S$ - a quantum analogue of the Hamilton-Jacobi functional [12], written as

$$
S_{\kappa}:=\int_{-\infty}^{+\infty} d t\left(p_{t} \dot{x}_{t}-\kappa(t) H_{t}\right)
$$

where $\kappa(t)$ is an appropriate test function.

The first term of the action operator $S_{\kappa}$ Eq. (14) is identical to the kinematical part of the classical phase space action functional. This 'Liouville' operator is formally written as

$$
V:=\int_{-\infty}^{\infty} d t\left(p_{t} \dot{x_{t}}\right)
$$

so that

$$
S_{\kappa}=V-H_{\kappa} .
$$

The 'average-energy' operator

$$
\hat{H}_{\kappa}=\int_{-\infty}^{\infty} d t \kappa(t) \hat{H}_{t} ; H_{t}:=\frac{p_{t^{2}}}{2 m}+\frac{m \omega^{2}}{2} x_{t}^{2}
$$


is also smeared in time by smearing functions $\kappa(t)$. The Hamiltonian operator may be employed (here for the special case $\kappa(t)=1)$ to define Heisenberg picture operators for the smeared operators like $x_{f}$

$$
\hat{x}_{f}(s):=e^{\frac{i}{\hbar} s \hat{H}} \hat{x}_{f} e^{-\frac{i}{\hbar} s \hat{H}}
$$

where $f=f(t)$ is a smearing function.

Hence $\hat{H}_{\kappa}$ generates transformations with respect to the Heisenberg picture parameter $s$, therefore, $s$ is the time label as it appears in the implementation of dynamical laws

$$
e^{\frac{i}{\hbar} \tau \hat{H}} \hat{x}_{f}(s) e^{-\frac{i}{\hbar} \tau \hat{H}}=\hat{x}_{f}(s+\tau) .
$$

The novel feature in this construction is the definition of the 'Liouville' operator $\hat{V}$, which is the quantum analogue of the kinematical term in the classical phase space action functional. The Liouville operator generates transformations with respect to the time label $t$ - as it appears in the history algebra, hence, $t$ the label of temporal logic or the label of kinematics

$$
e^{\frac{i}{\hbar} \tau \hat{V}} \hat{x}_{f}(s) e^{-\frac{i}{\hbar} \tau \hat{V}}=\hat{x}_{f^{\prime}}(s), \quad f^{\prime}(t)=f(t+\tau) .
$$

We must emphasise here the distinction between the notion of time evolution from that of logical time-ordering. The latter refers to the temporal ordering of logical propositions in the consistent histories formalism. The corresponding parameter $t$ does not coincide with the notion of physical time as it is measured for instance by a clock. It is an abstraction, which keeps from physical time only its ordering properties, namely that it designates the sequence at which different events happen-the same property that is kept by the notion of time-ordered product in quantum field theory. Making this distinction about time, it is natural to assume that in the HPO histories one may not use the same label for the time evolution of physical systems and the time-ordering of events. The former concept incorporates also the notion of a clock, namely it includes a measure of time duration, as something distinct from temporal ordering.

The realisation of this idea on the notion of time was possible in this particular framework because of the logical structure of the theory, as it was originally introduced in the consistent histories formalism and as it was later recovered as temporal logic in the HPO scheme.

One may say then that the definition of these two operators, $V$ and $H$, implementing time translations, signifies the distinction between the kinematics and the dynamics of the theory.

However a crucial result of the theory is that $\hat{S}_{\kappa}$ is the physical generator of the time translations in histories theory, as we can see from the way it appears in the decoherence functional and hence the physical predictions of the theory.

\section{CLASSICAL HISTORIES}

The HPO scheme and especially the history group suggests a reformulation of classical mechanics in the language of histories, which will prove very fruitful in the case of general relativity.
We consider the space of classical histories $\Pi=\{\gamma \mid \gamma: \mathbb{R} \rightarrow$ $\Gamma\}$ as paths on the single-time classical phase space $\Gamma$. We equip the history space with a symplectic structure $t \rightarrow\left(x_{t}, p_{t}\right)$ corresponding to the following Poisson brackets

$$
\begin{aligned}
\left\{x_{t}, x_{t^{\prime}}\right\} & =0 \\
\left\{p_{t}, p_{t^{\prime}}\right\} & =0 \\
\left\{x_{t}, p_{t^{\prime}}\right\} & =\delta\left(t, t^{\prime}\right)
\end{aligned}
$$

where

$$
\begin{aligned}
x_{t}: \Pi & \rightarrow \mathbb{R} \\
\gamma & \mapsto x_{t}(\gamma):=x(\gamma(t)) .
\end{aligned}
$$

The classical Hamilton equations may be written in terms of the Liouville function $V$ and the smeared Hamiltonian function $H$, which are the classical analogues of the corresponding operators we defined for the quantum theory

$$
\left\{F_{t}, V\right\}_{\Pi}\left(\gamma_{c l}\right)=\left\{F_{t}, H\right\}_{\Pi}\left(\gamma_{c l}\right),
$$

where

$$
V(\gamma):=\int d t p_{t} \dot{x}_{t}, \quad\left\{F_{t}, V\right\}=\dot{F}_{t}
$$

It follows the important conclusion that the solutions to the classical equations of motion are the specific paths that remain invariant under the symplectic transformations generated by the action $S$ for all functions $F_{t}$

$$
\{F, S\}_{\Pi}\left(\gamma_{c l}\right)=0
$$

where $S=V-H$. The Eq. (17) is essentially the histories analogue of the least action principle.

\section{GENERAL RELATIVITY HISTORIES FORMALISM}

Next we study the HPO formalism in the case of general relativity $[8,9]$. We show that the novel temporal structure of HPO - that distinguishes between the kinematics and the dynamics of a theory - suggests a spacetime description that is immediately related to the canonical one.

Let us consider a 4-manifold $M$ with topology $\Sigma \times R$, for a three-manifold $\Sigma$. We define the covariant history space

$$
\Pi^{\text {cov }}=T^{*} \operatorname{LRiem}(M)
$$

as the cotangent bundle of the space of all Lorentzian, globally hyperbolic four metrics on $\mathrm{M}$ and where $\operatorname{LRiem}(M)$ is the space of all Lorentzian four-metrics.

$\Pi^{c o v}$ is equipped with a symplectic structure with symplectic form $\Omega$

$$
\Omega=\int d^{4} X \delta \pi^{\mu v}(X) \wedge \delta g_{\mu v}(X)
$$

where $X \in M, g_{\mu \nu} \in L \operatorname{Riem}(M)$ and $\pi^{\mu \nu}$ its 'conjugate' variable and where $\delta g_{\mu v}$ is a one-form on $\Pi^{c o v}$ and $\delta$ represents the exterior derivative. 
Or else with the covariant Poisson brackets algebra, on $\Pi^{c o v}$

$$
\begin{aligned}
& \left\{g_{\mu v}(X), g_{\alpha \beta}\left(X^{\prime}\right)\right\}=0=\left\{\pi^{\mu v}(X), \pi^{\alpha \beta}\left(X^{\prime}\right)\right\} \\
& \left\{g_{\mu v}(X), \pi^{\alpha \beta}\left(X^{\prime}\right)\right\}=\delta_{(\mu v)}^{\alpha \beta} \delta^{4}\left(X, X^{\prime}\right),
\end{aligned}
$$

where $\delta_{(\mu v)}{ }^{\alpha \beta}:=\frac{1}{2}\left(\delta_{\mu}{ }^{\alpha} \delta_{v}{ }^{\beta}+\delta_{\mu}{ }^{\beta} \delta_{v}{ }^{\alpha}\right)$.

The physical meaning of $\pi$ can be understood after the $3+1$ decomposition of $M$ in which it will be related to the canonical conjugate momenta.

\section{A. The representation of the group $\operatorname{Diff}(M)$.}

The relation between the group of spacetime diffeomorphisms $\operatorname{Diff}(M)$ and the Dirac algebra of constraints has been an important matter of discussion in quantum gravity. We have showed that in this formalism of general relativity there exists a representation of the group of spacetime diffeomorphisms together with the Dirac algebra of constraints.

$\Pi^{\text {cov }}$ carries a symplectic action of the $\operatorname{Diff}(M)$ group, with generator defined for any vector field $W$ on $M$

$$
V_{W}:=\int d^{4} X \pi^{\mu v}(X) \mathcal{L}_{W} g_{\mu v}(X)
$$

where $\mathcal{L}_{W}$ denotes the Lie derivative with respect to $W$.

The functions $V_{W}$ satisfy the Lie algebra of $\operatorname{Diff}(M)$

$$
\left\{V_{W_{1}}, V_{W_{2}}\right\}=V_{\left[W_{1}, W_{2}\right]}
$$

where $\left[W_{1}, W_{2}\right]$ is the Lie bracket between vector fields $W_{1}$ and $W_{2}$ on the manifold $M$.

\section{B. Relation between spacetime and canonical description}

Next we study the relation between the covariant description and the standard canonical one.

We must emphasise here that the spacetime description we presented is kinematical - in the sense that we do not start from a Lagrangian formalism and from this deduce the canonical constraints. We rather start from the histories canonical general relativity and we show that this formalism is augmented by a spacetime description that carries a representation of the spacetime $\operatorname{Diff}(\mathrm{M})$ group.

In the standard canonical formalism we introduce a spacelike foliation $\mathcal{E}: \mathbb{R} \times \Sigma \rightarrow M$ on $M$, with respect to a fixed Lorentzian four-metric $g$. Then the spacelike character of the foliation function implies that the pull-back of the four metric on a surface $\Sigma$ is a Riemannian metric with signature +++ . In the histories theory we obtain a path of such Riemannian metrics $t \mapsto h_{i j}(t, \underline{x})$ each one defined on a copy of $\Sigma_{t}$ with the same $t$ label.

However a foliation cannot be spacelike with respect to all metrics $g$ and in general, for an arbitrary metric $g$ the pullback of a metric $\mathcal{E}^{*} g$ is not a Riemannian metric on $\Sigma$.

This point reflects a major conceptual problem of quantum gravity: the notion of 'spacelike' has no a priori meaning in a theory in which the metric is a non-deterministic dynamical variable; in absence of deterministic dynamics, the relation between canonical and covariant variables appears rather puzzling. In classical general relativity this is not a problem because 'spacelikeness' refers to the metric that solves the equations of motion. In quantum gravity however where one expects metric fluctuations the notion of spacelikeness is problematic.

In histories theory this problem is addressed by introducing the notion of a metric dependent foliation $\mathcal{E}[g]$, defined as a map $\mathcal{E}[g]: \operatorname{LRiem}(M) \mapsto F o l M$, that assigns to each Lorentzian metric a foliation that is always spacelike with respect to that metric. Then we use the metric dependent foliation $\mathcal{E}[g]$ to define the canonical decomposition of the metric $g$ with respect to the canonical three-metric $h_{i j}$, the lapse function $N$ and the shift vector $N^{i}$ as

$$
\begin{aligned}
h_{i j}(t, \underline{x}) & :=\mathcal{E}_{, i}^{\mu}(t, \underline{x} ; g] \mathcal{E}_{, j}^{v}(t, \underline{x} ; g] g_{\mu v}(\mathcal{E}(t, \underline{x} ; g]) \\
N_{i}(t, \underline{x}) & :=\mathcal{E}_{, i}^{\mu}(t, \underline{x} ; g] \dot{\mathcal{E}}^{v}(t, \underline{x} ; g] g_{\mu v}(\mathcal{E}(t, \underline{x} ; g]) \\
N^{2}(t, \underline{x}) & :=\dot{\mathcal{E}}^{\mu}(t, \underline{x} ; g] \dot{\mathcal{E}}^{v}(t, \underline{x} ; g] g_{\mu v}(\mathcal{E}(t, \underline{x} ; g])-N_{i} N^{i}
\end{aligned}
$$

Defined in this way $h_{i j}$ is always a Riemannian metric, with the correct signature.

In the histories theory therefore, the $3+1$ decomposition preserves the spacetime character of the canonical variables, a feature that we may expect to hold in a theory of quantum gravity.

\section{Relation between $\Pi^{c o v}$ and $\Pi^{c a n}$}

With the introduction of the metric-dependent foliation we can then write the symplectic form $\Omega$, on the space of canonical general relativity histories description $\Pi^{c a n}$, (using an equivalent canonical form of $\Omega$ ),

$$
\begin{aligned}
\Omega & =\int d^{4} X \delta \pi^{\mu v} \wedge \delta g_{\mu v} \\
& =\int d^{3} x d t\left(\delta \pi^{i j} \wedge \delta h_{i j}+\delta p \wedge \delta N+\delta p_{i} \wedge \delta N^{i}\right),
\end{aligned}
$$

by introducing conjugate momenta for the three-metric $\pi^{i j}$, the lapse function $p$ and the shift vector $p_{i}$.

Thus we prove the equivalence of the covariant history space $\Pi^{\text {cov }}=\left(T^{*} \operatorname{LRiem}(M)\right.$ with the space of paths on the canonical phase space of general relativity

$$
\Pi^{\text {can }}=\times_{t}\left(T^{*} \operatorname{Riem}\left(\Sigma_{t}\right) \times T^{*} \operatorname{Vec}\left(\Sigma_{t}\right) \times T^{*} C^{\infty}\left(\Sigma_{t}\right)\right),
$$

where $\operatorname{Riem}\left(\Sigma_{t}\right)$ is the space of all Riemannian three-metrics on the surface $\Sigma_{t}, \operatorname{Vec}\left(\Sigma_{t}\right)$ is the space of all vector fields on $\Sigma_{t}$, and $C^{\infty}\left(\Sigma_{t}\right)$ is the space of all smooth scalar functions on $\Sigma_{t}$.

\section{Canonical description}

The canonical history space of general relativity $\Pi^{c a n}$ is the Cartesian product of the cotangent bundles of the space of 
Riemannian three-metrics $\operatorname{Riem} \Sigma_{t}$, the space of vector fields on $\Sigma$ and the space of all scalar functions on $\Sigma$. Hence, $\Pi^{\text {can }}$ is a suitable subset of the Cartesian product of copies of the phase space $\Gamma$ of standard canonical general relativity

$$
\Pi^{\text {can }} \subset \times_{t} \Gamma_{t}, \quad \Gamma_{t}=\Gamma\left(\Sigma_{t}\right) .
$$

A history therefore is any smooth map

$$
t \mapsto\left(h_{i j}(t, \underline{x}), \pi^{k l}(t, \underline{x}), N^{i}(t, \underline{x}), p_{i}(t, \underline{x}), N(t, \underline{x}), p(t, \underline{x})\right) .
$$

We obtain the history version of the canonical Poisson brackets from the covariant Poisson brackets

$$
\begin{aligned}
\left\{h_{i j}(t, \underline{x}), \pi^{k l}\left(t^{\prime}, \underline{x}^{\prime}\right)\right\} & =\delta_{(i j)}{ }^{k l} \delta\left(t, t^{\prime}\right) \delta^{3}\left(\underline{x}, \underline{x}^{\prime}\right) \\
\left\{N(t, \underline{x}), p\left(t^{\prime}, \underline{x}^{\prime}\right)\right\} & =\delta\left(t, t^{\prime}\right) \delta^{3}\left(\underline{x}^{\prime}, \underline{x}^{\prime}\right) \\
\left\{N^{i}(t, \underline{x}), p_{j}\left(t^{\prime}, \underline{x}^{\prime}\right)\right\} & =\delta_{j}^{i} \delta\left(t, t^{\prime}\right) \delta^{3}\left(\underline{x}^{\prime}, \underline{x}^{\prime}\right) \\
\left\{h_{i j}(t, \underline{x}), h_{k l}\left(t^{\prime}, \underline{x}^{\prime}\right)\right\} & =0=\left\{\pi^{i j}(t, \underline{x}), \pi^{k l}\left(t^{\prime}, \underline{x}^{\prime}\right)\right\} \\
\left\{N(t, \underline{x}), N\left(t^{\prime}, \underline{x}^{\prime}\right)\right\} & =0=\left\{p(t, \underline{x}), p\left(t^{\prime}, \underline{x}^{\prime}\right)\right\} \\
\left\{N^{i}(t, \underline{x}), N^{j}\left(t^{\prime}, \underline{x}^{\prime}\right)\right\} & =0=\left\{p_{i}(t, \underline{x}), p_{j}\left(t^{\prime}, \underline{x}^{\prime}\right)\right\}
\end{aligned}
$$

where we have defined $\delta_{(i j)}{ }^{k l}:=\frac{1}{2}\left(\delta_{i}{ }^{k} \delta_{j}{ }^{l}+\delta_{i}{ }^{l} \delta_{j}{ }^{k}\right)$. All quantities $N, N^{i}, p$ and $p_{i}$ have vanishing Poisson brackets with $\pi^{i j}$ and $h_{i j}$.

\section{E. Invariance transformations of covariant and canonical descriptions}

The generators of the diffeomorphism group Diff(M), defined as Eq. (18), act on the spacetime or covariant variables in a natural way, generating spacetime diffeomorphisms

$$
\begin{aligned}
& \left\{g_{\mu v}(X), V_{W}\right\}=\mathcal{L}_{W} g_{\mu v}(X) \\
& \left\{\pi^{\mu v}(X), V_{W}\right\}=\mathcal{L}_{W} \pi^{\mu v}(X) .
\end{aligned}
$$

The coexistence of the spacetime and the canonical variables allows one to write the history analogue of the canonical constraints. The canonical description leads naturally to a one-parameter family of super-hamiltonians $t \mapsto \mathcal{H}_{\perp}(t, \underline{x})$ and super-momenta $t \mapsto \mathcal{H}_{i}(t, \underline{x})$,

$$
\begin{aligned}
\mathcal{H}_{\perp}(t, \underline{x}) & :=\kappa^{2} h^{-1 / 2}(t, \underline{x})\left(\pi^{i j}(t, \underline{x}) \pi_{i j}(t, \underline{x})\right. \\
& \left.-\frac{1}{2}\left(\pi_{i}^{i}\right)^{2}(t, \underline{x})\right)-\kappa^{-2} h^{1 / 2}(t, \underline{x}) R(t, \underline{x}) \\
\mathcal{H}^{i}(t, \underline{x}) & :=-2 \nabla_{j} \pi^{i j}(t, \underline{x}),
\end{aligned}
$$

where $\kappa^{2}=\frac{8 \pi G}{c^{2}}$ and the nabla $\nabla$ denotes the covariant derivative. We prove that they satisfy a history version of the Dirac algebra.

We may also write the constraints in a covariant form:

$$
\begin{aligned}
\mathcal{H}[\vec{L}] & =\int d^{4} X(\bar{E} \pi)^{\mu v} \mathcal{L}_{L} g_{\mu v}+2 \int d^{4} X(\bar{E} \pi)^{\mu v} n_{\mu} n^{\rho} \mathcal{L}_{L} g_{\rho v} \\
\mathcal{H}_{\perp}[L] & =\int d^{4} X\left[\kappa^{2} \frac{N}{\sqrt{-g}} \frac{1}{2} G_{\mu v \rho \sigma}(\bar{E} \pi)^{\mu \nu}(\bar{E} \pi)^{\rho \sigma_{-}} \kappa^{-2} \frac{\sqrt{-g}}{N}{ }^{3} R(h)\right] \\
\Phi(k) & =\int d^{4} X(\bar{E} \pi)^{\mu v} n_{\mu}(X ; g] k_{v}(X)
\end{aligned}
$$

where $\vec{L}^{\mu}(X ; g) n_{\mu}(X ; g]=0$ and $G_{\mu v \rho \sigma}$ is a covariant expression of the Dewitt metric. The supermomentum $\mathcal{H}[\vec{L}]$ is smeared with a horizontal vector field $\mathcal{L}$, normal to the foliation vector normal to the leaves $n^{\mu}$; the superhamiltonian $\mathcal{H}_{\perp}[L]$ is smeared with a scalar function $L$; while the primary constraints $p=p^{i}=0$ are smeared together in a compact form, of the constraint $\Phi(k)$, by a one-form $k_{v}$. $E$ is a kernel function that appears first when we relate the spacetime variables with the canonical ones; when the foliation does not depend on the metric $E$ it equals the unit operator.

\section{F. Equivariance condition}

In order to study the explicit relation between the $\operatorname{Diff}(\mathrm{M})$ group and the canonical constraints, we introduce an important mathematical restriction on the foliation, the equivariance condition.

The equivariance condition follows from the requirement of general covariance, namely that the description of the theory ought to be invariant under changes of coordinate systems implemented by spacetime diffeomorphisms.

A metric-dependent foliation functional

$$
\mathcal{E}: \operatorname{LRiem}(M) \rightarrow \operatorname{Fol}(M)
$$

is defined as an equivariant foliation if it satisfies the simple mathematical condition

$$
\mathcal{E}\left[f^{*} g\right]=f^{-1} \circ \mathcal{E}[g],
$$

for all Lorentzian metrics $g$ and $f \in \operatorname{Diff}(M)$.

The interpretation of the condition Eq. (23) is as follows: if we perform a change of the coordinate system of the theory under a spacetime diffeomorphism, then the expressions of the objects defined in it will change, and so the foliation functional $\mathcal{E}[g]$ and the four-metric $g$ will also change. Then, the change of the foliation due to the change of the coordinate system must be compensated by the change due to its functional dependence on the metric $g$. This is essentially the passive interpretation of the spacetime diffeomorphisms. Loosely speaking what we have achieved with the introduction of the equivariance condition is that the foliation functional 'looks the same' in all coordinate systems.

The physical requirement is that the change of any tensor field $A(\cdot, g]$, associated to the foliation, under a diffeomorphism $f$ is compensated by the change due to its functional dependence on $g$. Hence, if we consider a diffeomorphism transformation $f$, and we denote its pull-back operation by $f^{*}$, the equivariance condition is given by the expression

$$
\left(f^{*} A\right)(\cdot, g]=A\left(\cdot, f^{*} g\right]
$$

\section{G. Relation between the invariance groups}

One of the deepest issues to be addressed in canonical gravity is the relation of the algebra of constraints to the spacetime 
diffeomorphisms group. The canonical constraints depend on the $3+1$ decomposition and hence on the foliation functional.

The equivariance condition manifests a striking result both in its simplicity and its implications: the action of the diffeomorphisms group Diff $(\mathrm{M})$ preserves the set of the constraints, in the sense that it transforms a constraint into another of the same type but of different argument. Hence, the choice of an equivariance foliation implements that histories canonical field variables related by diffeomorphisms are physically equivalent

$$
\begin{aligned}
\left\{V_{W}, \Phi(k)\right\} & =\Phi\left(\mathcal{L}_{W} k\right) \\
\left\{V_{W}, \mathcal{H}(\vec{L})\right\} & =\mathcal{H}\left(\delta_{W} \vec{L}\right) \\
\left\{V_{W}, \mathcal{H}_{\perp}(L)\right\} & =\mathcal{H}_{\perp}\left(\mathcal{L}_{W} L\right) .
\end{aligned}
$$

Here $\delta_{W}$ is the total change due to a diffeomorphism that takes into account that $L^{\mu}$ is normal to $n^{\mu}$, which is itself metric dependent.

Furthermore, this result means also that, the group Diff(M) is represented in the space of the true degrees of freedom, the reduced phase space. We can say equivalently that the space of true degrees of freedom is invariant under $\operatorname{Diff}(\mathrm{M})$.

Hence, in the histories theory the requirement of the physical equivalence of different choices of time direction is satisfied by means of the equivariance condition.

\section{H. Reduced state space}

Finally we study the reduction procedure as implemented in the histories framework. General relativity is a parameterized system in the sense that it has vanishing Hamiltonian on the reduced phase space due to the presence of first class constraints.

One may define the history constraint surface $C_{h}=\{t \mapsto$ $C, t \in \mathbb{R}\}$ as the space of maps from the real line to the singletime constraint surface $C$ of canonical general relativity.

The history reduced state space is obtained as the quotient of the history constraint surface, with respect to the action of the constraints, i.e. the space of orbits on $C_{h}$ arising from the action of the constraints.

The histories Hamiltonian constraint is defined as

$$
H_{\kappa}=\int d t \kappa(t) h_{t}
$$

where $h_{t}:=h\left(x_{t}, p_{t}\right)$ is first-class constraint. For all values of the smearing function $\kappa(t)$, the history Hamiltonian constraint $H_{\mathrm{K}}$ generates canonical transformations on the history constraint surface.

It has been proved [8] that the history reduced state space $\Pi_{\text {red }}$ is a symplectic manifold that can be identified with the space of paths on the canonical reduced state space $\Gamma_{\text {red }}$ :

$$
\Pi_{\text {red }}=\left\{t \mapsto \Gamma_{\text {red }}, t \in \mathbb{R}\right\}
$$

We have proved therefore that the histories reduced state space is identical with the space of paths on the canonical reduced state space.
Consequently the time parameter $t$ also exists on $\Pi_{r e d}$, and the notion of time ordering remains on the space of the true degrees of freedom $\Pi_{r e d}$. This last result is in contrast to the standard canonical theory where there exists ambiguity with respect to the notion of time after reduction.

Moreover, the phase space action functional $S$

$S:=\int d t \int d^{3} \underline{x}\left\{\tilde{\pi}^{i j}(t, \underline{x}) \dot{h}_{i j}(t, \underline{x})+\tilde{p}_{i} \dot{N}^{i}+\tilde{p} \dot{N}-\mathcal{H}(N)-\mathcal{H}(\vec{N})\right\}$

commutes weakly with the constraints, so it can be projected on the histories reduced state space

$$
\left\{S, \Phi(k)+\mathcal{H}[\vec{L}]+\mathcal{H}_{\perp}[L]\right\} \simeq 0 .
$$

It then serves its role in determining the equations of motion, as we have shown in the theory of classical histories [15].

In order for a function on the full state space $\Pi$, to be a physical observable (i.e., to be projectable into a function on $\left.\Pi_{r e d}\right)$, it is necessary and sufficient that it commutes with the constraints on the constraint surface. Contrary to the canonical treatments of parameterised systems, the classical equations of motion are explicitly realised on the reduced state space $\Pi_{\text {red }}$.

Indeed, the equations of motion are the paths on the phase space that remain invariant under the symplectic transformations generated by the projected action

$$
\left\{\tilde{S}, F_{t}\right\}\left(\gamma_{c l}\right)=0, F_{t} \text { constant in } t
$$

where $\tilde{S}$ and $\tilde{V}$ are respectively the action and Liouville functions projected on $\Pi_{\text {red }}$.

The usual dynamical equations for the canonical fields $h_{i j}$ and $\pi^{i j}$ are equivalent to the history Poisson bracket equations

$$
\begin{aligned}
& \left\{S, h_{i j}(t, \underline{x})\right\}\left(\gamma_{c l}\right)=0 \\
& \left\{S, \pi^{i j}(t, \underline{x})\right\}\left(\gamma_{c l}\right)=0
\end{aligned}
$$

The path $\gamma_{c l}$ is a solution of the classical equations of motion, and therefore corresponds to a spacetime metric that is a solution of the Einstein equations.

The canonical action functional $S$ is also diffeomorphicinvariant

$$
\left\{V_{W}, S\right\}=0 \text {. }
$$

This is a significant result: it leads to the conclusion that the action functional and the equations of motion (25-26) are the 'observables' of general relativity theory, as has been indicated from the Lagrangian treatment of the theory. Hence, the dynamics of the histories theory is invariant under the group of spacetime diffeomorphisms.

It is important to remember that the parameter with respect to which the orbits of the constraints are defined, is not in any sense identified with the physical time $t$. In particular, one can distinguish the paths corresponding to the classical equations of motion by the condition

$$
\{F, \tilde{V}\}_{\gamma_{c l}}=0
$$


where $F$ is a functional of the field variables, and $\gamma_{c l}$ is a solution to the equations of motion.

In standard canonical theory, the elements of the reduced state space are all solutions to the classical equations of motion. In histories canonical theory, however, an element of the reduced state space is a solution to the classical equations of motion only if it also satisfies the condition Eq. (28). The reason for this is that the histories reduced state space $\Pi_{\text {red }}$ contains a much larger number of paths (essentially all paths on $\left.\Gamma_{\text {red }}\right)$. For this reason, histories theory may naturally describe observables that commute with the constraints but which are not solutions to the classical equations of motion.

This last point should be particularly emphasised, because of its possible corresponding quantum analogue. We know that in quantum theory, paths may be realised that are not solutions to the equations of motion. My belief is that the histories formalism will distinguish between instantaneous laws [16] (namely constraints), and dynamical laws (equations of motion). Hence, it is possible to have a quantum theory for which the instantaneous laws are satisfied, while the classical dynamical laws are not. This distinction is present, for example, in the history theory of the quantised electromagnetic field, where all physical states satisfy the Gauss law exactly, however electromagnetism field histories are possible which do not satisfy the dynamical equations, i.e., Maxwell's equations. For parameterised systems, this distinction is not possible within the canonical formalism, nevertheless as we explained, it does arise in the histories formalism.

The equations of motion (28) imply that physical observables have constant values on the solutions to the classical equations of motion. This need not be the case quantum mechanically, hence quantum realised paths need not be characterised by 'frozen' values of their physical parameters.

\section{NOTES ON QUANTIZATION}

These are significant results for developing a theory of quantum gravity. It indicates that the histories scheme can incorporate intrinsically the basic symmetry of general rela- tivity, namely general covariance - as manifested by the existence of a realisation of the $\operatorname{Diff}(\mathrm{M})$ group - and the invariance of the Hamiltonian constraints under its action.

Furthermore it provides a possible quantum gravity theory where the full Lorentzian metric may be quantised, unlike some spatial part of the metric of the canonical schemes. For this purpose we may follow the quantum algorithm we described in the beginning of this presentation. That is to seek a representation of the history algebra or the histories commutation relations that are defined with reference to the whole of spacetime and not just a 3-surface; in particular, these history variables include a quantised Lorentzian spacetime metric. Of course problems of defining properly quantum Hamiltonian operators still remains in a first estimation of the formalism.

Another possible direction to follow is to develop a histories analogue of loop quantum gravity, as this is a successful canonical theory in many respects. In the canonical treatment, the basic algebra is defined with reference to objects that have support on loops in the three-dimensional surface $\Sigma$. The natural object in the histories description is the $S L(2, \mathbf{C})$ ) connection. An obvious first step would be to write the kinematical Hilbert space based on a representation of an $S L(2, C)$ connection on $M$ instead of the $S U(2)$ connection on $\Sigma$.

This is a more complicated matter, and there is no guarantee that there exists a correspondence between the histories $S L(2, \mathbf{C})$ theory and the canonical $S U(2)$ one. The major difference is that the $\operatorname{SL}(2, \mathbf{C})$ group is non-compact, hence the definition of the proper Hilbert space cannot follow the steps of the canonical construction.

The mathematical structures of a quantisation based on histories will conceivably be very different from those in the canonical theory. For this reason, the history construction may uncover substantially different properties from those that arise in the existing approaches to loop quantum gravity.

\section{ACKNOWLEDGMENTS}

This work was supported by a Marie Curie Reintegration Grant of the European Commission.
[1] R.B. Griffiths, Consistent Histories and the Interpretation of Quantum Mechanics, J. Stat. Phys. 36, 219 (1984); R. Omnès, Logical Reformulation of Quantum Mechanics: I Foundations, J. Stat. Phys. 53, 893 (1988); The Interpretation of Quantum Mechanics (Princeton University Press, Princeton, 1994).

[2] M. Gell-Mann and J.B. Hartle, Quantum mechanics in the Light of Quantum Cosmology, in: Complexity, Entropy and the Physics of Information, W.H. Zurek (ed.) (Addison Wesley, Reading, 1990); Classical Equations for Quantum Systems, Phys. Rev. D47, 3345 (1993); J.B. Hartle, Spacetime quantum mechanics and the quantum mechanics of spacetime, in: Proceedings on the 1992 Les Houches School,Gravitation and Quantisation (1993).

[3] C.J. Isham, Quantum logic and the histories approach to quantum theory, J. Math. Phys. 35, 2157 (1994).

[4] C.J. Isham and N. Linden, Quantum temporal logic and de- coherence functionals in the histories approach to generalised quantum theory, J. Math. Phys. 35, 5452 (1994).

[5] C.J. Isham and N. Linden, Continuous histories and the history group in generalised quantum theory, J. Math. Phys. 36, 5392 (1995).

[6] C. Isham, N. Linden, K. Savvidou and S. Schreckenberg, Continuous time and consistent histories, J. Math. Phys. 37, 2261 (1998).

[7] K. Savvidou, The action operator in continuous time histories, J. Math. Phys. 40, 5657 (1999).

[8] K. Savvidou, General relativity histories theory II: Invariance groups, Class. Quant. Grav. 21, 631 (2004).

[9] K. Savvidou, General relativity histories theory I: The spacetime character of the canonical description, Class. Quant. Grav. 21, 615 (2004).

[10] K. Savvidou, Poincaré invariance for continuous-time histories, 
J. Math. Phys. 43, 30532002.

[11] C.J. Isham and K.N. Savvidou, Quantising the Foliation in History Quantum Field Theory, J. Math. Phys. 43 (2003).

[12] P.A.M. Dirac, The Lagrangian in quantum mechanics, in: Selected papers on quantum electrodynamics, J. Schwinger (ed.) (Dover Publications, New York, 1958).

[13] A. Burch, Histories Electromagnetism, J. Math. Phys. 45(6), 2153 (2004).

[14] K. Kuchar, The problem of time in canonical quantization, in: A. Ashtekar \& J. Stachel (eds.), Conceptual Problems of Quantum Gravity (Birkhäuser, Boston), pp. 141-171.

[15] We need to remember that in classical histories the analogue of a physical observable is a one-parameter family of functions that correspond to single-time physical observables. For a oneparameter family of functions $F_{t}$ that commutes with the constraints the equations of motion yield that $F_{t}$ is constant - something that is expected in the classical theory.

[16] A thorough analysis on the connection between instantaneous laws (Gauss' theorema egregium) and the dynamical laws of general relativity is presented by Kuchar in [14], where he deduces Einstein's equations starting from the (instantaneous) geometric description of gravity. 